\title{
ACE Inhibitory and Antihypertensive Activities of Wine Lees and Relationship among Bioactivity and Phenolic Profile
}

\author{
Raúl López-Fernández-Sobrino (D), Jorge R. Soliz-Rueda (D), Maria Margalef (D), Anna Arola-Arnal (D), \\ Manuel Suárez $\mathbb{D}$, Francisca I. Bravo *iD and Begoña Muguerza (D)
}

check for updates

Citation: López-Fernández-Sobrino, R.; Soliz-Rueda, J.R.; Margalef, M.; Arola-Arnal, A.; Suárez, M.; Bravo, F.I.; Muguerza, B. ACE Inhibitory and Antihypertensive Activities of Wine Lees and Relationship among Bioactivity and Phenolic Profile. Nutrients 2021, 13, 679. https:// doi.org/10.3390/nu13020679

Academic Editor:

Benno Zimmermann

Received: 20 January 2021

Accepted: 16 February 2021

Published: 20 February 2021

Publisher's Note: MDPI stays neutral with regard to jurisdictional claims in published maps and institutional affiliations.

Copyright: (c) 2021 by the authors. Licensee MDPI, Basel, Switzerland. This article is an open access article distributed under the terms and conditions of the Creative Commons Attribution (CC BY) license (https:/ / creativecommons.org/licenses/by/ $4.0 /)$.
Nutrigenomics Research Group, Department of Biochemistry and Biotechnology, Universitat Rovira i Virgili, 43007 Tarragona, Spain; raul.lopez@urv.cat (R.L.-F.-S.); jorgericardo.soliz@urv.cat (J.R.S.-R.); maria.margalef.jornet@gmail.com (M.M.); anna.arola@urv.cat (A.A.-A.); manuel.suarez@urv.cat (M.S.); begona.muguerza@urv.cat (B.M.)

* Correspondence: franciscaisabel.bravo@urv.cat; Tel.: +34-97-755-88-37
Abstract: Wine lees (WL) are by-products generated in the winemaking process. The aim of this study was to investigate the angiotensin-converting enzyme inhibitory (ACEi) activity, and the blood pressure (BP) lowering effect of WL from individual grape varieties. The relationship among their activities and phenolic profiles was also studied. Three WL, from Cabernet, Mazuela, and Garnacha grape varieties, were firstly selected based on their ACEi properties. Their phenolic profiles were fully characterized by UHPLC-ESI-Q-TOF-MS. Then, their potential antihypertensive effects were evaluated in spontaneously hypertensive rats (SHR). BP was recorded before and after their oral administrations $(2,4,6,8,24$, and $48 \mathrm{~h}$ ) at a dose of $5 \mathrm{~mL} / \mathrm{kg}$ bw. Cabernet WL (CWL) exhibited a potent antihypertensive activity, similar to that obtained with the drug Captopril. This BP-lowering effect was related to the high amount of anthocyanins and flavanols present in these lees. In addition, a potential hypotensive effect of CWL was discarded in normotensive Wistar-Kyoto rats. Finally, the ACEi and antihypertensive activities of CWL coming from a different harvest were confirmed. Our results suggest the potential of CWL for controlling arterial BP, opening the door to commercial use within the wine industry.

Keywords: blood pressure; Cabernet grape variety; hypertension; polyphenols; SHR; winery by-product

\section{Introduction}

Nowadays, the leading cause of death worldwide is cardiovascular disease (CVD), being hypertension (HTN) one of their major risk factors [1]. The global prevalence of HTN is high since it is suffered by one in four adults [2]. In fact, a $25 \%$ reduction of its prevalence is one of the global targets to be attained by 2025 [3]. The adoption of healthy lifestyles in combination with pharmacological therapy has been shown to be effective for controlling blood pressure (BP) and improving CVD [4]. In this sense, angiotensin-converting enzyme (ACE) inhibitors, such as Captopril or Enalapril, are the first-choice treatments for HTN [5]. These drugs act blocking the ACE, which plays an important role in the BP regulation within the renin-angiotensin system [6]. In fact, its inhibition exerts a clear BP-lowering effect since ACE catalyzes the hydrolysis of the peptide angiotensin I (Ang I) to generate the vasoconstrictor Ang II. ACE is also involved in the kallikrein-kinin system, degrading the vasodilator bradykinin. Despite the effectiveness in controlling the BP of ACE inhibitors, new natural compounds are being investigated since drugs can cause certain side effects in some patients [7]. These alternatives could result in the reduction of HTN at the early or mid-stages of the disease [8]. Thus, antihypertensive compounds from natural sources have emerged as an excellent alternative to synthetic drugs, and are highly demanded, and researched.

Agri-food by-products have emerged as a novel source to obtain these natural antihypertensive agents since they can contain compounds with a wide range of biological 
properties [9]. The use of these waste products as a source of bioactive compounds allows their revaluation, making the food and agricultural industries more sustainable and environmentally friendly [10,11]. Grapes are one of the world's largest fruit crops and the wine production process generates large amounts of by-products [12]. Some winery by-products, such as grape seeds and skin, have already been used as a source for the extraction of phenolic compounds with numerous health benefits, including antihypertensive properties [13-17]. However, the presence of antihypertensive compounds in other winery by-products as wine lees $(\mathrm{WL})$ remains unexplored.

According to the Council Regulation (EEC) No. 337/79, WL are "the residue that forms at the bottom of recipients containing wine, after fermentation, during storage or after authorized treatments, as well as the residue obtained following the filtration or centrifugation of this product" [18]. The potential application in the food, cosmetics, and pharmaceutical industries of WL has been suggested [19]. In fact, some studies have reported antioxidant, antimicrobial, anti-inflammatory, and cardioprotective properties of WL [19-21]. Differently from other winemaking by-products, WL have been least studied and exploited, and only the extraction of ethanol and tartaric acid is performed on a large scale [22]. However, WL could present ACEi and/or antihypertensive properties since their use as a source of phenolic compounds has been suggested [20]. In this sense, some studies have detected anthocyanins, flavonols, flavanols, and phenolic acids in WL [19,23-26].

Therefore, the aim of this study was to investigate the potential antihypertensive effect of WL. Thus, the ACEi activity was evaluated in five different WL generated from the winemaking process using single grape varieties. Three of these WL were selected according to their ACEi activity. Their phenolic profile was fully characterized and their antihypertensive activities were tested in spontaneously hypertensive rats (SHR). In addition, we evaluated BP-lowering effect of the selected WL in normotensive rats Wistar-Kyoto (WKY) to rule out a potential hypotensive effect. Furthermore, their ACEi and antihypertensive activities were evaluated using WL from a different harvest.

\section{Materials and Methods}

\subsection{Chemicals and Reagents}

Human Angiotensin-converting enzyme (ACE, EC 3.4.15.1, 5.1 U/mg), Captopril (PubChem CID: 44093) and N-Hippuryl-His-Leu (Hip-His-Leu), were purchased from Santa Cruz Biotechnology (Dallas, TX, USA). O-aminobenzoylglicil-p-nitrofenilalanilprolina (o-Abz-Gly-p-Phe(NO2)-Pro-OH, PubChem CID: 128860) was provided by Bachem Feinchemikalien (Bubendorf, Switzerland). Acetonitrile and trifluoroacetic acid HPLC grade were purchased from Sigma-Aldrich (Madrid, Spain). Gallic acid, (-)-epicatechin, pcoumaric acid, and (+)-catechin were purchased from Fluka/Sigma-Aldrich; chlorogenic acid, caffeic acid, malvidin-3-O-glucoside, (-)-epigallocatechin gallate, and procyanidin dimer B2 were purchased from Extrasynthése (Lyon, France); cyanidin-3-O-rutinoside was purchased from PhytoLab (Vestenbergsgreuth, Germany); resveratrol was purchased from Carl Roth (Karlsruhe, Germany); and rutin was kindly provided by Nutrafur S.A. (Murcia, Spain). All other chemical solvents used were of analytical grade.

\subsection{Wine Lees}

WL were provided by Grandes Vinos y Viñedos, S.A located in the Cariñena P.O.D area (Zaragoza, Spain). They were collected after racking the wines. All the wines were elaborated with a single grape variety and following the same manufacturing procedure. The selected grape varieties were Cabernet, Garnacha, Mazuela, Merlot (all of them red grape varieties), and Macabeo (white grape variety). Moreover, lees obtained in the elaboration of wine with Cabernet grapes were supplied from two different harvests (CWL and CWL2). WL were centrifuged at $3000 \times \mathrm{g}$ for $15 \mathrm{~min}$ at $4{ }^{\circ} \mathrm{C}$ to remove solid particles. Supernatants were collected and kept at $4{ }^{\circ} \mathrm{C}$ until their analysis or administration to animals. 


\subsection{Measurement of the ACEi Activity}

ACEi activity was measured by a fluorescence technique according to Mas-Capdevila et al. [27]. This technique is based on the ability of ACE to hydrolyze the fluorescence compound o-Abz-Gly-p-Phe $\left(\mathrm{NO}_{2}\right)-\mathrm{Pro}-\mathrm{OH}$. Inhibition of this enzyme produces a decrease in fluorescence values. Thus, an aliquot of $40 \mu \mathrm{L}$ of WL was added to a microtiter-plate well and mixed with $160 \mu \mathrm{L}$ of $0.45 \mathrm{mM}$ o-Abz-Gly-p-Phe $\left(\mathrm{NO}_{2}\right)-\mathrm{Pro}-\mathrm{OH}$ dissolved in $150 \mathrm{mM}$ Tris-base buffer ( $\mathrm{pH} 8.3$ ), containing $1.125 \mathrm{M} \mathrm{NaCl}$. The enzymatic reaction started by adding $40 \mu \mathrm{L}$ of an ACE solution prepared in $0.15 \mathrm{M}$ Tris buffer (pH 8.3) containing $0.1 \mu \mathrm{M}$ of $\mathrm{ZnCl}_{2}$ (enzyme concentration in the well was $0.04 \mathrm{U} / \mathrm{mL}$ ). The reaction was carried out at $37^{\circ} \mathrm{C}$ during $30 \mathrm{~min}$. At this time point, fluorescence measurements using $\lambda$ ex $360 \mathrm{~nm}$ and $\lambda \mathrm{em} 400 \mathrm{~nm}$ were recorded and used to determine the inhibitory activity. The ACEi activity was calculated using the following formula:

$$
\text { ACEi activity }(\%): 1-\frac{\mathrm{S}-\mathrm{Bs}}{\mathrm{PC}-\mathrm{B}} \times 100
$$

where $S$ is the fluorescence emitted after the action of ACE on the substrate, with inhibitor (sample), Bs is the fluoresce emitted by the substrate and the sample, Pc is the fluoresce emitted after the action of ACE on the substrate, without inhibitor, and B is the fluoresce emitted by the substrate and the sample.

ACEi activity was expressed as a percentage $(\%)$ or $\mathrm{IC}_{50}(\mu \mathrm{L})$. Percentage of ACEi activity was determined at $\mathrm{WL}$ volume of $0.16 \mu \mathrm{L}$ in order to compare the effects of different $\mathrm{WL}$ on $\mathrm{ACE}$ activity. $\mathrm{IC}_{50}$ was calculated by linear approximation regression. Data are represented as the mean value of three determinations \pm SD.

\subsection{Detection and Quantification of the Phenolic Compounds from Wine Lees}

The individual phenolic profile of WL from Garnacha, Cabernet, and Mazuela WL was carried out by high-performance liquid chromatography coupled to electrospray ionisation and quadrupole time-of-flight mass spectrometry (UHPLC-ESI-Q-TOF-MS). WL samples were diluted twice with water:methanol with $1 \%$ of formic acid $(50: 50, \mathrm{v}: \mathrm{v})$, centrifuged for $5 \mathrm{~min}$ at $17,150 \times \mathrm{g}$ at room temperature and supernatants were directly analyzed using a 1290 UHPLC Infinity II series coupled to a Q-TOF/MS 6550 (Agilent Technologies, Palo Alto, CA, USA). Two different methodologies based on UHPLC-ESI-Q-TOF-MS systems were used to separate, detect, and quantify the non-anthocyanin and anthocyanin phenolic compounds. For the separation of non-anthocyanin compounds, an Acquity HSST3 C18 column (150 $\mathrm{mm} \times 2.1 \mathrm{~mm}$ i.d., $1.8 \mu \mathrm{m}$ particle size) (Waters, Milford, MA, USA) was used and the mobile phase consisted of (A) water:acetic acid (95:5, v:v) and (B) acetonitrile. The gradient mode was as follows: initial conditions, $0 \% \mathrm{~B} ; 0-0.5 \mathrm{~min}, 0 \% \mathrm{~B} ; 0.5-18 \mathrm{~min}$, $0-30 \% \mathrm{~B} ; 18-21 \mathrm{~min}, 30-95 \% \mathrm{~B} ; 21-24 \mathrm{~min}, 95 \% \mathrm{~B}$; and $24-25 \mathrm{~min}, 100-0 \%$ B. A post-run of $6 \mathrm{~min}$ was required for column re-equilibration. The flow rate was set at $0.550 \mathrm{~mL} / \mathrm{min}$ and column temperature was $45^{\circ} \mathrm{C}$. The injection volume was $2.5 \mu \mathrm{L}$ for all runs. Electrospray ionization (ESI) operating in negative mode was conducted with a gas temperature at $200{ }^{\circ} \mathrm{C}$ and the flow rate was $14 \mathrm{~L} / \mathrm{min}$. Nebulizer gas pressure was $20 \mathrm{psi}$, sheath gas temperature was $350^{\circ} \mathrm{C}$, sheath gas flow was $11 \mathrm{~L} / \mathrm{min}$, and the capillary voltage was $3000 \mathrm{~V}$. The anthocyanins compounds were separated on an Acquity BEH C18 column $(100 \mathrm{~mm} \times 2.1 \mathrm{~mm}, 1.7 \mu \mathrm{m}$ particle size) (Waters) and the mobile phase consisted on water:formic acid $(9: 1, \mathrm{v}: \mathrm{v})(\mathrm{A})$ and acetonitrile (B). The gradient mode was as follows: initial conditions, $0 \% \mathrm{~B} ; 0-0.5 \mathrm{~min}, 0 \% \mathrm{~B} ; 0.5-5 \mathrm{~min}, 0-9 \% \mathrm{~B} ; 5-7 \mathrm{~min}, 9-15 \% \mathrm{~B} ; 7-9.5 \mathrm{~min}$, $15-30 \% \mathrm{~B} ; 9.5-10 \mathrm{~min}, 30-100 \% \mathrm{~B} ; 10-12 \mathrm{~min}, 100 \% \mathrm{~B}$; and $12-12.1 \mathrm{~min}, 100-0 \% \mathrm{~B}$. A postrun of $5 \mathrm{~min}$ was required for column re-equilibration. The flow rate was set at $0.4 \mathrm{~mL} / \mathrm{min}$ and column temperature was $25^{\circ} \mathrm{C}$. The injection volume was $2.5 \mu \mathrm{L}$ for all runs. ESI operating in positive mode was conducted with a gas temperature set at $200{ }^{\circ} \mathrm{C}$ and the flow rate was $14 \mathrm{~L} / \mathrm{min}$. Nebulizer gas pressure was $20 \mathrm{psi}$, sheath gas temperature was $350{ }^{\circ} \mathrm{C}$, sheath gas flow was $11 \mathrm{~L} / \mathrm{min}$ and the capillary voltage was $3000 \mathrm{~V}$. The mass spectra were recorded between $100-1000 \mathrm{~m} / \mathrm{z}$ at $2.5 \mathrm{spectra} / \mathrm{s}$ for both methodologies. 
The assignment of the phenolic compounds was performed by direct comparison with the commercial standards available or by bibliographic information using chromatographic behavior, mass accurate molecular ion ([M-H]- or $[\mathrm{M}-\mathrm{H}]+)$, and fragmentation patterns $[19,28,29]$. The obtained calibration curves of commercial standards available were used for the quantification of their corresponding phenolic compounds. When commercial standards were not available, a tentative quantification was carried out by using the calibration curve of the standard more similar.

\subsection{Experimental Procedure in Rats}

Male SHR and WKY rats (17-20-week-old, weighing 310-350 g) were purchased from Charles River Laboratories España S.A. (Barcelona, Spain). The animals were housed at a temperature of $23{ }^{\circ} \mathrm{C}$ with $12 / 12 \mathrm{~h}$ light/dark cycles and $50 \%$ of humidity. After quarantine and a training period of 2 weeks, animals were given tap water and a standard diet (A04 Panlab, Barcelona, Spain) ad libitum during the experiments. The initial values of the systolic blood pressure (SBP) and diastolic blood pressure (DBP) in the SHR were $186.6 \pm 1.7$ and $153.6 \pm 2.6 \mathrm{mmHg}$, respectively.

Figure 1 shows a graphical representation of the three experimental designs used in this study. A first study was carried out in SHR in order to evaluate antihypertensive effect of three WL obtained in the winemaking process with three different grapes varieties: Cabernet, Garnacha, and Mazuela (Figure 1A). For that, a single dose of $5 \mathrm{~mL} / \mathrm{kg}$ bw of the WL was administered to SHR rats. Water and Captopril (50 mg/kg bw, dissolved in water) were used as a negative and positive control, respectively. A second study was carried out in WKY rats to discard a possible hypotensive effect of the Cabernet WL (CWL, Figure 1B). These WL were administered to animals in a single dose $(5 \mathrm{~mL} / \mathrm{kg} \mathrm{bw})$. Water was used as a negative control. In both studies, SBP and DBP were recorded in the animals before and 2, 4, $6,8,24$, and $48 \mathrm{~h}$ after treatment administration to rats using the tail-cuff method, according to Quiñones et al. [30]. $\triangle \mathrm{SBP}$ and $\triangle \mathrm{DBP}$ were calculated as the difference between the mean values of SBP or DBP after and before treatment administration for each rat. Data were expressed as the mean values \pm SEM for a minimum of six experiments.
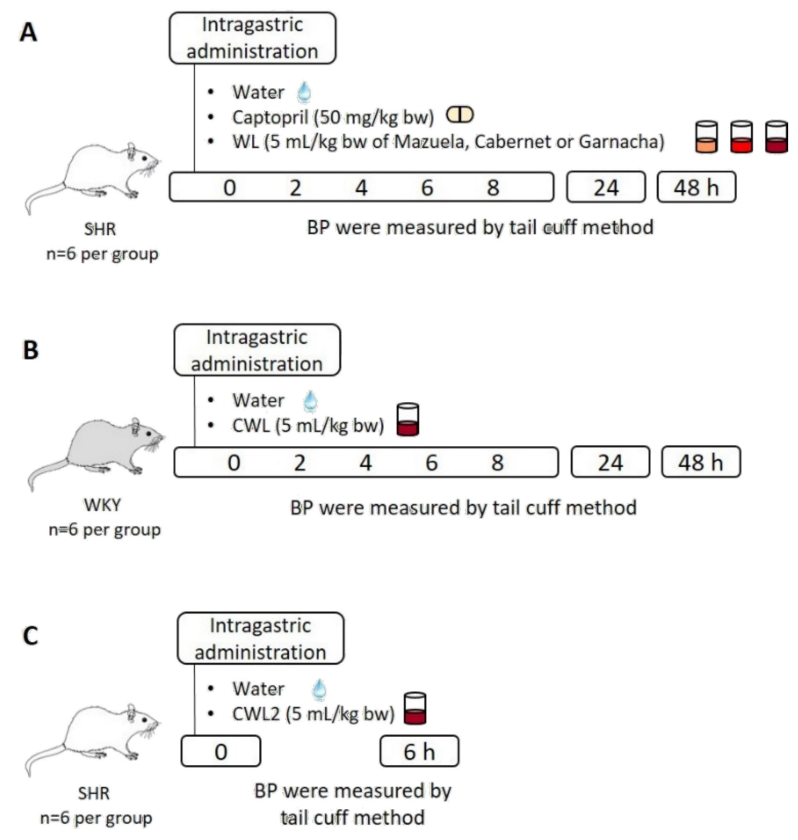

Figure 1. Graphical representation of the three in vivo studies carried out to investigate (A) the effect of three wine lees (WL) on blood pressure (BP) in spontaneously hypertensive rats (SHR), (B) the effect of Cabernet WL (CWL) on BP in normotensive Wistar-Kyoto rats (WKY) and (C) the effect of CWL from a different harvest (CWL2) at $6 \mathrm{~h}$ post-administration in SHR. 
For the evaluation of the effect of different harvests of CWL on the decrease in BP, an additional trial was conducted with SHR (Figure 1C). The study was carried out by administering a dose of $5 \mathrm{~mL} / \mathrm{kg}$ bw of CWL2 to SHR ( $n=6$ per group). Water was used as a negative control. BP was recorded before and $6 \mathrm{~h}$ after administration.

In all the in vivo studies, treatments were administered by gastric intubation between 9 and 10 am in a volume between 1.5 and $2 \mathrm{~mL}$ by oral gavage.

All animal protocols followed in this study were approved by the Animal Ethics Review Committee for Animal Experimentation of the Universitat Rovira i Virgili and further approved by Generalitat de Catalunya (permission number 10780).

\subsection{Statistical Analysis}

BP differences produced by the administration of the different WL were analyzed by a two-way analysis of variance (ANOVA) for the studies with SHR and WKY rats. Student's T-test was used to evaluate differences between CWL from different campaigns in both $\mathrm{IC}_{50}$ analysis and antihypertensive study. A one-way ANOVA was used to evaluate differences between phenolic compounds in WL. All the analyses were performed using GraphPad Prism 7.04 for Windows (GraphPad Software, San Diego, California). Outliers were determined by using Grubbs' test. Differences between groups were considered significant when $p<0.05$.

\section{Results}

\subsection{Selection of the Wine Lees}

Table 1 shows the ACEi activity of the five WL used in this study. As it is shown, WL obtained from red grape varieties (Cabernet, Garnacha, Mazuela, and Merlot) showed a greater ACEi activity than the one related to the white grape variety (Macabeo). Specifically, red grapes showed a percentage of ACEi activity between $28 \%$ and $56 \%$. In addition, the concentration of WL needed to inhibit $50 \%$ of the ACE activity $\left(\mathrm{IC}_{50}\right)$ was also determined. They ranged between $0.15 \pm 0.01$ and $3.74 \pm 0.05 \mu \mathrm{L}$. Cabernet, Garnacha, and Mazuela WL were the ones with the highest activities (lower than $0.5 \mu \mathrm{L}$, Table 1) and were selected for further studies. The dose-response of ACE inhibition of some of the tested WL is represented in Figure 2.

Table 1. Angiotensin-converting enzyme inhibitory (ACEi) activity of the wine lees obtained in the winemaking process using different individual grape varieties.

\begin{tabular}{ccc}
\hline \multirow{2}{*}{ Grape Variety } & \multicolumn{2}{c}{ ACEi activity } \\
\cline { 2 - 3 } & $\mathbf{\%}^{*}$ & IC $_{\mathbf{5 0}}(\boldsymbol{\mu L})$ \\
\hline Cabernet & $55.69 \pm 1.92$ & $0.15 \pm 0.01$ \\
Garnacha & $44.16 \pm 2.54$ & $0.22 \pm 0.01$ \\
Mazuela & $50.70 \pm 8.10$ & $0.21 \pm 0.03$ \\
Merlot & $28.76 \pm 0.34$ & $0.32 \pm 0.02$ \\
Macabeo & $<10$ & $3.74 \pm 0.05$ \\
\hline
\end{tabular}

${ }^{*} \mathrm{ACEi}$ activity showed by a wine lees volume of $0.16 \mu \mathrm{L}$.

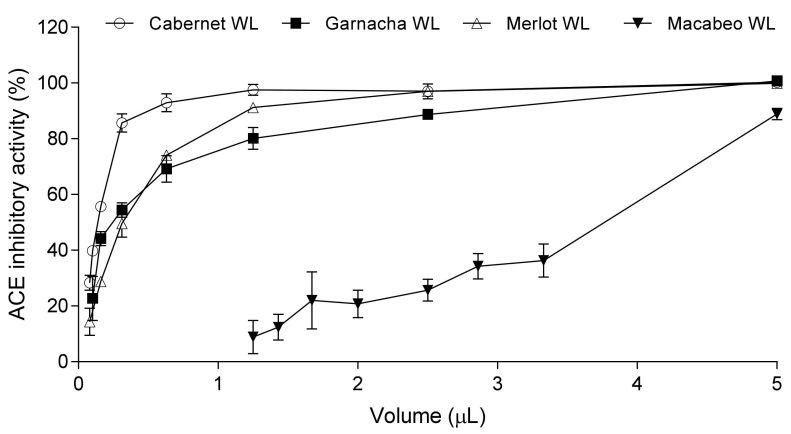

Figure 2. Dose-response curves (effect as a function of the dose in $\mu \mathrm{L}$ ) for Cabernet, Garnacha, Merlot, and Macabeo wine lees (WL). Values are the average of three replicates \pm SD. 


\subsection{Determination of the Phenolic Profile of the Three Selected Wine Lees}

Phenolic composition of the selected WL was determined using commercial standards. As standards of all the phenolic compounds were not always available, a tentative quantification of these other compounds was carried out using the calibration curve of the most similar available structures. Figure 3 shows the results of the overlapped extract ion chromatograms (EIC) of non-anthocyanin phenolic compounds analyzed by UHPLC-(ESI)-Q-TOF-MS (Figure 3A) and anthocyanin phenolic compounds analyzed by UHPLC-(ESI + )-Q-TOF-MS (Figure 3B). Table 2 shows the total phenolic content and total content of flavanols, flavonols, phenolic acids, stilbenes, and anthocyanins of Cabernet, Mazuela, and Garnacha WL. The total content of phenolic compounds in the CWL was almost double than the content measured in Mazuela and Garnacha WL $(690.6,395.3$, and $379.6 \mathrm{mg} / \mathrm{L}$, respectively). In addition, the contribution of the different phenolic families to the total phenolic content was different depending on the type of WL. Flavanols was the main family in the CWL distantly followed by anthocyanins and phenolic acids $(311.1,153.5$, and $133.5 \mathrm{mg} / \mathrm{L}$, respectively). However, flavanols and phenolic acids, in the same proportion, were the main groups in Mazuela and Garnacha WL. The main difference found between CWL and both Mazuela and Garnacha WL was the highest content of flavanols and anthocyanins showed by CWL (Table 2).

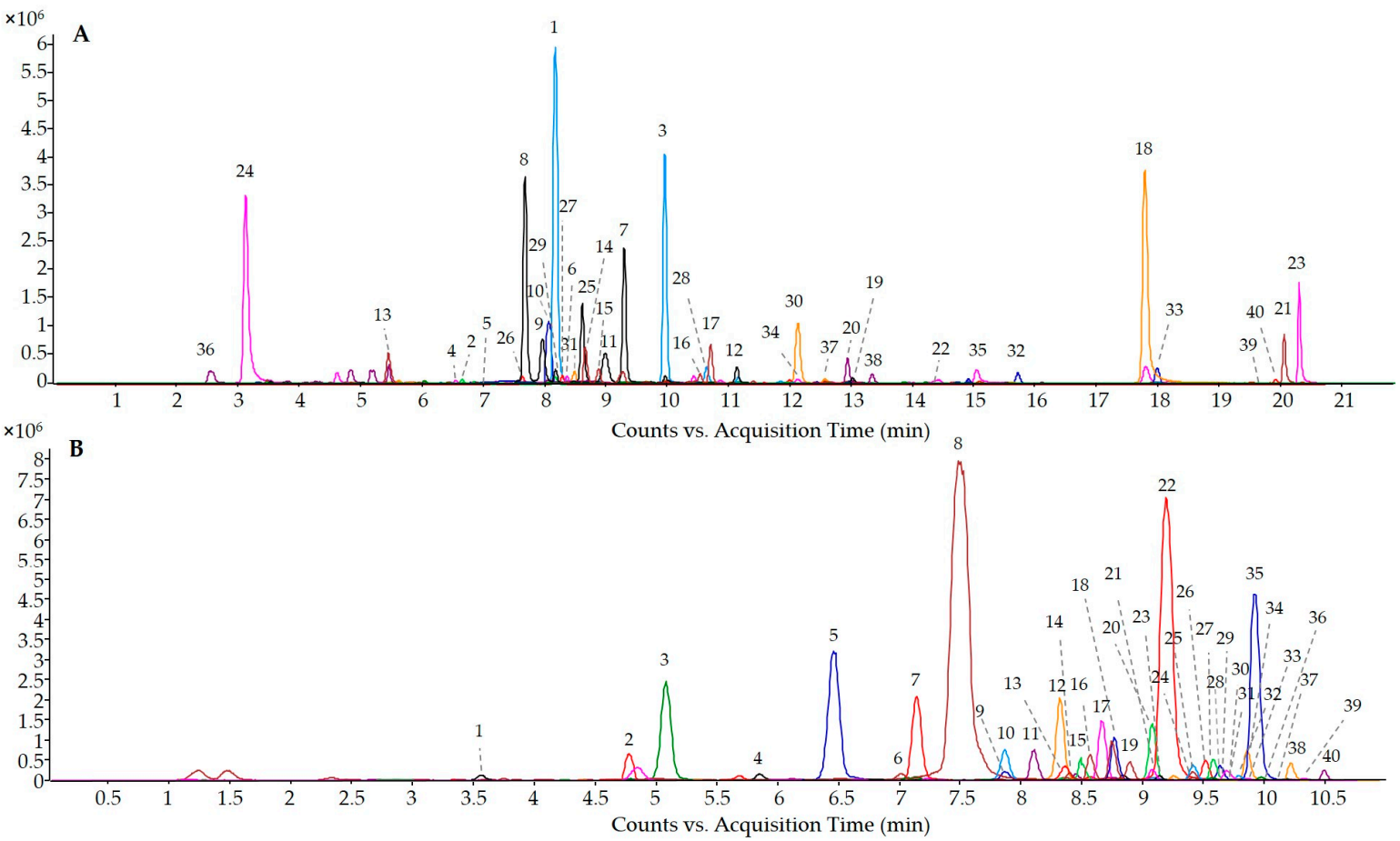

Figure 3. Overlapped extract ion chromatograms (EIC) of (A) non-anthocyanin wine lees (WL)-phenolic compounds analyzed by UHPLC-(ESI-)-Q-TOF-MS and (B) anthocyanin WL-phenolic compounds analyzed by UHPLC-(ESI + )-Q-TOFMS. Chromatographic peaks are numbered according to Tables 3 and 4 .

Table 2. Total composition of flavanols, flavonols, phenolic acids, stilbenes, and anthocyanins in studied wine lees (WL).

\begin{tabular}{cccc}
\hline Phenolic Compounds & Cabernet WL $\mathbf{( m g / L )}$ & Mazuela WL $\mathbf{( m g} / \mathbf{L})$ & Garnacha WL $\mathbf{~ ( m g / L ) ~}$ \\
\hline Flavanols & 331.11 & 122.63 & 154.60 \\
Flavonols & 57.62 & 44.83 & 57.28 \\
Phenolic acids & 133.54 & 132.75 & 103.05 \\
Stilbenes & 14.73 & 20.08 & 13.59 \\
Anthocyanins & 153.53 & 74.99 & 51.12 \\
\hline Total & 690.63 & 395.28 & 379.64 \\
\hline
\end{tabular}


Table 3. Characterization of phenolic compounds in Cabernet, Mazuela, and Garnacha wine lees (WL) by UHPLC-(ESI-)-QTOF-MS.

\begin{tabular}{|c|c|c|c|c|c|c|c|}
\hline & Compounds & R.T. (min) & [M-H]- & $\begin{array}{l}\text { Fragment } \\
(\mathrm{m} / \mathrm{z})\end{array}$ & $\begin{array}{l}\text { Cabernet WL } \\
(\mathrm{mg} / \mathrm{L})\end{array}$ & $\begin{array}{l}\text { Mazuela WL } \\
(\mathrm{mg} / \mathrm{L})\end{array}$ & $\begin{array}{l}\text { Garnacha } \\
\text { WL (mg/L) }\end{array}$ \\
\hline \multicolumn{8}{|c|}{ Flavanols } \\
\hline 1 & Catechin & 8.17 & 289.0718 & & $97.63 \pm 0.62^{\mathrm{a}}$ & $39.27 \pm 0.25^{b}$ & $56.65 \pm 0.36^{\mathrm{c}}$ \\
\hline 2 & Catechin gallate $^{1}$ & 6.66 & 441.0827 & 289.07209 & $0.80 \pm 0.01^{\mathrm{a}}$ & $1.53 \pm 0.03^{b}$ & $0.79 \pm 0.01^{\mathrm{a}}$ \\
\hline 3 & Epicatechin & 9.96 & 289.0718 & & $43.48 \pm 0.23^{a}$ & $12.94 \pm 0.07^{b}$ & $20.16 \pm 0.11^{b}$ \\
\hline 4 & (Epi)catechin O-glucoside iso ${ }^{2}$ & 6.55 & 451.1246 & 289.0721 & $0.50 \pm 0.00^{\mathrm{a}}$ & $0.50 \pm 0.00^{\mathrm{a}}$ & $0.90 \pm 0.00^{\mathrm{a}}$ \\
\hline 5 & (Epi)catechin O-glucoside iso ${ }^{2}$ & 7.41 & 451.1246 & 289.0721 & $0.33 \pm 0.00^{\mathrm{a}}$ & $0.29 \pm 0.00^{\mathrm{a}}$ & $0.43 \pm 0.00^{\mathrm{a}}$ \\
\hline 6 & (Epi)catechin O-glucoside iso ${ }^{2}$ & 8.37 & 451.1246 & 289.0721 & $1.47 \pm 0.03^{\mathrm{a}}$ & $0.77 \pm 0.01^{\mathrm{b}}$ & $1.64 \pm 0.03^{a}$ \\
\hline 7 & Procyanidin dimer B2 & 9.30 & 577.1387 & 289.0733 & $34.60 \pm 0.01^{\mathrm{a}}$ & $9.61 \pm 0.00^{b}$ & $9.09 \pm 0.00^{\mathrm{c}}$ \\
\hline 8 & Procyanidin dimer iso $1^{3}$ & 7.68 & 577.1387 & 289.0733 & $64.21 \pm 0.34^{\mathrm{a}}$ & $32.11 \pm 0.17^{\mathrm{b}}$ & $32.37 \pm 0.17^{b}$ \\
\hline 9 & Procyanidin dimer iso $2^{3}$ & 7.97 & 577.1387 & 289.0733 & $14.26 \pm 0.09^{a}$ & $4.19 \pm 0.03^{b}$ & $5.81 \pm 0.04^{\mathrm{c}}$ \\
\hline 10 & Procyanidin dimer iso $3^{3}$ & 8.18 & 577.1387 & 289.0733 & $2.96 \pm 0.02^{\mathrm{a}}$ & $0.84 \pm 0.01^{b}$ & $1.50 \pm 0.01^{\mathrm{c}}$ \\
\hline 11 & Procyanidin dimer iso $4^{3}$ & 8.99 & 577.1387 & 289.0733 & $12.80 \pm 0.00^{\mathrm{a}}$ & $2.71 \pm 0.00^{\mathrm{b}}$ & $3.79 \pm 0.00^{\mathrm{c}}$ \\
\hline 12 & Procyanidin dimer iso $5^{3}$ & 11.14 & 577.1387 & 289.0733 & $4.32 \pm 0.04^{\mathrm{a}}$ & $1.54 \pm 0.02^{b}$ & $1.97 \pm 0.02^{b}$ \\
\hline 13 & Procyanidin trimer iso ${ }^{3}$ & 5.46 & 865.2016 & 577.1369 & $16.28 \pm 0.29^{a}$ & $5.14 \pm 0.09^{b}$ & $7.91 \pm 0.14^{\mathrm{c}}$ \\
\hline 14 & Procyanidin trimer iso $2^{3}$ & 8.67 & 865.2016 & 577.1369 & $14.35 \pm 0.71^{\mathrm{a}}$ & $4.62 \pm 0.23^{b}$ & $5.15 \pm 0.25^{c}$ \\
\hline 15 & Procyanidin trimer iso ${ }^{3}$ & 8.89 & 865.2016 & 577.1369 & $6.11 \pm 0.03^{\mathrm{a}}$ & $2.37 \pm 0.01^{b}$ & $2.55 \pm 0.01^{\mathrm{b}}$ \\
\hline 16 & Procyanidin trimer iso $4^{3}$ & 10.55 & 865.2016 & 577.1369 & $3.22 \pm 0.14^{\mathrm{a}}$ & $1.38 \pm 0.06^{\mathrm{b}}$ & $1.19 \pm 0.06^{b}$ \\
\hline 17 & Procyanidin trimer iso $5^{3}$ & 10.71 & 865.2016 & 577.1369 & $13.79 \pm 0.23^{\mathrm{a}}$ & $2.82 \pm 0.05^{b}$ & $2.70 \pm 0.05^{b}$ \\
\hline \multicolumn{8}{|c|}{ Flavonols } \\
\hline 18 & Quercetin & 17.80 & 301.0372 & & $36.78 \pm 0.17^{a}$ & $28.62 \pm 0.13^{b}$ & $25.35 \pm 0.12^{c}$ \\
\hline 19 & Quercetin-3-O-glucoside ${ }^{4}$ & 13.00 & 463.0904 & 301.0361 & $1.63 \pm 0.04^{\mathrm{a}}$ & $2.73 \pm 0.08^{b}$ & $7.68 \pm 0.21^{\mathrm{c}}$ \\
\hline 20 & Quercetin-3-O-glucuronide 4 & 12.95 & 477.0702 & 301.0369 & $2.42 \pm 0.01^{\mathrm{a}}$ & $5.62 \pm 0.03^{b}$ & $4.86 \pm 0.02^{\mathrm{c}}$ \\
\hline 21 & Kaempferol ${ }^{4}$ & 20.07 & 285.0405 & & $5.15 \pm 0.02^{\mathrm{a}}$ & $1.63 \pm 0.01^{b}$ & $9.35 \pm 0.04^{\mathrm{c}}$ \\
\hline 22 & kaempferol-3-O-glucuronide 4 & 14.22 & 461.0763 & 285.0412 & $0.48 \pm 0.01^{\mathrm{a}}$ & $1.31 \pm 0.02^{b}$ & $3.24 \pm 0.05^{\mathrm{c}}$ \\
\hline 23 & Isorhamnetin ${ }^{4}$ & 20.31 & 315.0531 & & $11.16 \pm 0.12^{\mathrm{a}}$ & $4.92 \pm 0.05^{\mathrm{b}}$ & $6.80 \pm 0.07^{c}$ \\
\hline \multicolumn{8}{|c|}{ Phenolic acids } \\
\hline 24 & Gallic acid & 3.13 & 169.0193 & & $120.87 \pm 3.67^{a}$ & $121.19 \pm 3.68^{\mathrm{a}}$ & $96.11 \pm 2.92^{b}$ \\
\hline 25 & Caffeic acid & 8.63 & 179.0401 & & $3.27 \pm 0.04^{\mathrm{a}}$ & $2.18 \pm 0.02^{\mathrm{a}}$ & $1.09 \pm 0.01^{\mathrm{a}}$ \\
\hline 26 & Caffeic acid O-glucoside iso ${ }^{5}$ & 7.64 & 341.0878 & 179.0350 & $0.55 \pm 0.02^{\mathrm{a}}$ & $1.21 \pm 0.05^{\mathrm{a}}$ & $0.13 \pm 0.01^{\mathrm{a}}$ \\
\hline 27 & Caffeic acid O-glucoside iso ${ }^{5}$ & 8.29 & 341.0878 & 179.0350 & $0.66 \pm 0.03^{a}$ & $1.13 \pm 0.05^{\mathrm{a}}$ & $0.20 \pm 0.01^{\mathrm{a}}$ \\
\hline 28 & p-Coumaric acid & 10.65 & 163.0439 & & $3.44 \pm 0.03^{\mathrm{a}}$ & $3.73 \pm 0.04^{\mathrm{a}}$ & $1.15 \pm 0.01^{\mathrm{a}}$ \\
\hline 29 & 4-Hydroxybenzoic acid & 8.17 & 137.0243 & & $1.67 \pm 0.05^{\mathrm{a}}$ & $0.89 \pm 0.03^{a}$ & $1.19 \pm 0.04^{\mathrm{a}}$ \\
\hline 30 & Ferulic acid & 12.00 & 193.0506 & & $0.75 \pm 0.01^{\mathrm{a}}$ & $0.27 \pm 0.00^{\mathrm{a}}$ & $0.51 \pm 0.01^{\mathrm{a}}$ \\
\hline 31 & Vanillic acid & 8.51 & 167.0350 & & $2.33 \pm 0.07^{\mathrm{a}}$ & $2.15 \pm 0.08^{a}$ & $2.67 \pm 0.04^{\mathrm{a}}$ \\
\hline \multicolumn{8}{|c|}{ Stilbenes } \\
\hline 32 & trans-Resveratrol $^{6}$ & 15.73 & 227.0714 & & $4.60 \pm 0.02^{\mathrm{a}}$ & $3.63 \pm 0.02^{b}$ & $3.12 \pm 0.01^{\mathrm{c}}$ \\
\hline 33 & Resveratrol iso $1^{6}$ & 18.00 & 227.0714 & & $2.95 \pm 0.01^{\mathrm{a}}$ & $2.56 \pm 0.01^{b}$ & $0.97 \pm 0.00^{c}$ \\
\hline 34 & Resveratrol O-glucoside iso ${ }^{6}$ & 12.44 & 389.1242 & 227.0721 & $0.27 \pm 0.00^{\mathrm{a}}$ & $1.22 \pm 0.02^{b}$ & $1.17 \pm 0.02^{b}$ \\
\hline 35 & Resveratrol O-glucoside iso ${ }^{6}$ & 14.92 & 389.1242 & 227.0721 & $1.35 \pm 0.02^{\mathrm{a}}$ & $6.01 \pm 0.10^{b}$ & $3.32 \pm 0.05^{\mathrm{c}}$ \\
\hline 36 & Piceatannol 6 & 2.59 & 243.0663 & 203.0727 & $4.20 \pm 0.05^{\mathrm{a}}$ & $4.96 \pm 0.06^{b}$ & $4.04 \pm 0.05^{\mathrm{c}}$ \\
\hline 37 & Piceatannol 3-O-glucoside iso ${ }^{6}$ & 12.89 & 405.1208 & 243.0670 & $0.22 \pm 0.01^{\mathrm{a}}$ & $0.14 \pm 0.00^{\mathrm{b}}$ & $0.04 \pm 0.00^{\mathrm{c}}$ \\
\hline 38 & Piceatannol 3-O-glucoside iso ${ }^{6}$ & 13.15 & 405.1208 & 243.0670 & $0.06 \pm 0.00^{\mathrm{a}}$ & $0.18 \pm 0.00^{\mathrm{b}}$ & $0.05 \pm 0.00^{\mathrm{a}}$ \\
\hline 39 & Viniferin-iso ${ }^{6}$ & 19.53 & 453.1344 & 116.9291 & $0.27 \pm 0.01^{\mathrm{a}}$ & $0.33 \pm 0.01^{\mathrm{a}}$ & $0.15 \pm 0.00^{b}$ \\
\hline 40 & Viniferin-iso ${ }^{6}$ & 19.92 & 453.1344 & 116.9291 & $0.81 \pm 0.02^{\mathrm{a}}$ & $1.05 \pm 0.03^{b}$ & $0.73 \pm 0.02^{\mathrm{c}}$ \\
\hline
\end{tabular}

Abbreviations: Retention time (R.T.). Wine lees (WL). ${ }^{a, b, c}$ Different letters indicate significant differences in the content of each individual phenolic compound between the different WL $\left(p<0.05\right.$; one-way ANOVA) ${ }^{1}$ Tentatively quantified using the catechin calibrating curve.

2 Tentatively quantified using the epicatechin calibrating curve. ${ }^{3}$ Tentatively quantified using the procyanidin dimer B2 calibrating curve.

${ }^{4}$ Tentatively quantified using the quercetin calibrating curve. ${ }^{5}$ Tentatively quantified using the caffeic acid calibrating curve. ${ }^{6}$ Tentatively quantified using the resveratrol calibrating curve. 
Table 4. Characterization of anthocyanin in Cabernet, Mazuela, and Garnacha wine lees (WL) by UHPLC- (ESI +)-Q-TOF-MS.

\begin{tabular}{|c|c|c|c|c|c|c|c|}
\hline & Anthocyanins & R.T. (min) & {$[\mathrm{M}-\mathrm{H}]+$} & $\underset{(m / z)}{\text { Fragment }}$ & $\begin{array}{c}\text { Cabernet WL } \\
(\mathrm{mg} / \mathrm{L})\end{array}$ & $\begin{array}{c}\text { Mazuela WL } \\
(\mathrm{mg} / \mathrm{L})\end{array}$ & $\begin{array}{l}\text { Garnacha } \\
\text { WL (mg/L) }\end{array}$ \\
\hline 1 & Gallocatechin-Malvidin-3-glucoside dimer ${ }^{1}$ & 3.58 & 797.2035 & & $0.25 \pm 0.01^{\mathrm{a}}$ & $0.10 \pm 0.00^{\mathrm{a}}$ & $0.16 \pm 0.00^{\mathrm{a}}$ \\
\hline 2 & Malvidin-3-glucoside-(epi) catechin ${ }^{1}$ & 4.84 & 781.1974 & & $1.11 \pm 0.01^{\mathrm{a}}$ & $0.53 \pm 0.00^{b}$ & $0.50 \pm 0.00^{b}$ \\
\hline 3 & Delphinidin-3-glucoside ${ }^{2}$ & 5.06 & 465.1028 & 303.0511 & $3.69 \pm 0.04^{\mathrm{a}}$ & $2.98 \pm 0.03^{b}$ & $1.39 \pm 0.01^{c}$ \\
\hline 4 & Cyanidin-3-glucoside ${ }^{2}$ & 5.85 & 449.1078 & 287.0531 & $0.23 \pm 0.01^{\mathrm{a}}$ & $0.20 \pm 0.01^{\mathrm{a}}$ & $0.16 \pm 0.01^{\mathrm{a}}$ \\
\hline 5 & Petunidin-3-glucoside ${ }^{3}$ & 6.47 & 479.1184 & 317.0669 & $5.03 \pm 0.06^{\mathrm{a}}$ & $4.90 \pm 0.06^{\mathrm{a}}$ & $2.18 \pm 0.03^{b}$ \\
\hline 6 & Petunidin-3-glucoside-pyruvic acid ${ }^{3}$ & 7.05 & 547.1082 & 385.0547 & $0.09 \pm 0.00^{\mathrm{a}}$ & $0.06 \pm 0.00^{\mathrm{a}}$ & $0.03 \pm 0.00^{\mathrm{a}}$ \\
\hline 7 & Peonidin-3-glucoside ${ }^{3}$ & 7.14 & 463.1235 & 301.0717 & $2.72 \pm 0.04^{\mathrm{a}}$ & $1.83 \pm 0.03^{b}$ & $2.48 \pm 0.04^{\mathrm{c}}$ \\
\hline 8 & Malvidin-3-glucoside $^{1}$ & 7.48 & 493.1341 & 331.0843 & $60.67 \pm 0.68^{a}$ & $43.90 \pm 0.49^{b}$ & $26.78 \pm 0.30^{c}$ \\
\hline 9 & Peonidin-3-glucoside-pyruvic acid ${ }^{3}$ & 7.81 & 531.1133 & 369.0607 & $0.04 \pm 0.00^{\mathrm{a}}$ & $0.02 \pm 0.00^{\mathrm{a}}$ & $0.02 \pm 0.00^{a}$ \\
\hline 10 & Delphinidin-(6-acetyl)-3-glucoside ${ }^{2}$ & 7.87 & 507.1133 & 303.0496 & $0.91 \pm 0.02^{\mathrm{a}}$ & $0.09 \pm 0.00^{b}$ & $0.02 \pm 0.00^{\mathrm{b}}$ \\
\hline 11 & Visitin A (malvidin-3-glucoside-pyruvic acid) ${ }^{1}$ & 8.11 & 561.1239 & 399.0730 & $1.23 \pm 0.01^{\mathrm{a}}$ & $0.63 \pm 0.01^{b}$ & $0.35 \pm 0.00^{c}$ \\
\hline 12 & Visitin B (malvidin-3-glucoside-acetaldehyde) ${ }^{1}$ & 8.32 & 517.1341 & 355.0826 & $3.06 \pm 0.08^{a}$ & $4.92 \pm 0.12^{\mathrm{b}}$ & $5.11 \pm 0.00^{b}$ \\
\hline 13 & Malvidin-3-glucoside-ethyl-(epi) catechin ${ }^{1}$ & 8.40 & 809.2287 & & $0.37 \pm 0.00^{\mathrm{a}}$ & $0.09 \pm 0.00^{\mathrm{b}}$ & $0.31 \pm 0.13^{a}$ \\
\hline 14 & Cyanidin-(6-acetyl)-3-glucoside ${ }^{2}$ & 8.45 & 491.1184 & 491.1189 & $0.20 \pm 0.00^{\mathrm{a}}$ & $0.02 \pm 0.00^{\mathrm{b}}$ & $0.01 \pm 0.00^{b}$ \\
\hline 15 & Acetylvisitin $\mathrm{A}^{1}$ & 8.50 & 603.1344 & 399.0718 & $0.79 \pm 0.03^{a}$ & $0.10 \pm 0.00^{\mathrm{b}}$ & $0.15 \pm 0.00^{b}$ \\
\hline 16 & Malvidin-3-glucoside-ethyl-(epi) catechin ${ }^{1}$ & 8.57 & 809.2287 & & $1.38 \pm 0.02^{\mathrm{a}}$ & $0.51 \pm 0.01^{\mathrm{b}}$ & $1.65 \pm 0.00^{\mathrm{c}}$ \\
\hline 17 & Petunidin-(6-acetyl)-3-glucoside ${ }^{3}$ & 8.66 & 521.1378 & 317.0667 & $1.29 \pm 0.04^{\mathrm{a}}$ & $0.16 \pm 0.01^{b}$ & $0.04 \pm 0.02^{b}$ \\
\hline 18 & Malvidin-3-glucoside-ethyl-(epi) catechin ${ }^{1}$ & 8.75 & 809.2287 & & $2.04 \pm 0.06^{\mathrm{a}}$ & $0.80 \pm 0.03^{b}$ & $2.63 \pm 0.00^{c}$ \\
\hline 19 & Acetylvisitin B ${ }^{1}$ & 8.77 & 559.1446 & 355.0813 & $1.66 \pm 0.05^{\mathrm{a}}$ & $0.47 \pm 0.01^{\mathrm{b}}$ & $0.27 \pm 0.08^{b}$ \\
\hline 20 & Peonidin-(6-acetyl)-3-glucoside ${ }^{3}$ & 9.08 & 505.1341 & 301.0714 & $1.32 \pm 0.03^{\mathrm{a}}$ & $0.13 \pm 0.00^{\mathrm{b}}$ & $0.08 \pm 0.01^{b}$ \\
\hline 21 & Delphinidin-(6-coumaroyl)-3-glucoside ${ }^{2}$ & 9.08 & 611.1395 & 303.0508 & $0.44 \pm 0.01^{\mathrm{a}}$ & $0.55 \pm 0.01^{\mathrm{a}}$ & $0.09 \pm 0.00^{b}$ \\
\hline 22 & Malvidin-(6-acetyl)-3-glucoside ${ }^{1}$ & 9.13 & 535.1446 & 331.0836 & $28.39 \pm 0.03^{a}$ & $2.57 \pm 0.00^{b}$ & $0.79 \pm 0.00^{\mathrm{c}}$ \\
\hline 23 & Coumaroylvisitin $\mathrm{A}^{1}$ & 9.29 & 707.1607 & 399.0718 & $0.20 \pm 0.00^{\mathrm{a}}$ & $0.13 \pm 0.00^{\mathrm{b}}$ & $0.04 \pm 0.00^{b}$ \\
\hline 24 & Malvidin-(6-caffeoyl)-3-glucoside ${ }^{1}$ & 9.41 & 655.1657 & 331.0808 & $0.36 \pm 0.02^{\mathrm{a}}$ & $0.10 \pm 0.00^{\mathrm{b}}$ & $0.04 \pm 0.00^{\mathrm{b}}$ \\
\hline 25 & Cyanidin-(6-coumaroyl)-3-glucoside ${ }^{2}$ & 9.42 & 595.1446 & 287.0560 & $0.10 \pm 0.00^{\mathrm{a}}$ & $0.11 \pm 0.00^{\mathrm{a}}$ & $0.03 \pm 0.00^{b}$ \\
\hline 26 & Catechin-ethyl-Malvidin-3-acetylglucoside dimer ${ }^{1}$ & 9.43 & 851.2511 & & $0.88 \pm 0.03^{a}$ & $0.03 \pm 0.00^{\mathrm{b}}$ & $0.06 \pm 0.00^{b}$ \\
\hline 27 & Petunidin-(6-coumaroyl)-3-glucoside ${ }^{3}$ & 9.52 & 625.1552 & 317.0662 & $0.74 \pm 0.03^{\mathrm{a}}$ & $0.78 \pm 0.01^{\mathrm{a}}$ & $0.16 \pm 0.00^{b}$ \\
\hline 28 & Pinotin A (malvidin-3-glucoside-vinylcatechol) ${ }^{1}$ & 9.53 & 625.1552 & 463.0998 & $0.84 \pm 0.02^{\mathrm{a}}$ & $0.88 \pm 0.02^{\mathrm{a}}$ & $0.18 \pm 0.00^{b}$ \\
\hline 29 & Malvidin-glucoside-vinyl-catechin ${ }^{1}$ & 9.56 & 805.1974 & & $0.15 \pm 0.00^{\mathrm{a}}$ & $0.08 \pm 0.00^{\mathrm{b}}$ & $0.16 \pm 0.00^{\mathrm{a}}$ \\
\hline 30 & Coumaroylvisitin B ${ }^{1}$ & 9.58 & 663.1708 & 355.0822 & $0.91 \pm 0.03^{\mathrm{a}}$ & $1.08 \pm 0.04^{\mathrm{b}}$ & $1.12 \pm 0.04^{\mathrm{b}}$ \\
\hline 31 & Malvidin-3-glucoside-vinylguaiacol ${ }^{1}$ & 9.63 & 639.1708 & 331.0823 & $0.59 \pm 0.01^{\mathrm{a}}$ & $0.37 \pm 0.01^{\mathrm{b}}$ & $0.17 \pm 0.00^{b}$ \\
\hline 32 & Catechin-ethyl-malvidin-3-coumaroylglucoside dimer ${ }^{1}$ & 9.70 & 955.2785 & & $0.68 \pm 0.01^{\mathrm{a}}$ & $0.21 \pm 0.00^{\mathrm{b}}$ & $0.51 \pm 0.01^{\mathrm{a}}$ \\
\hline 33 & Catechin-ethyl-malvidin-3-acetylglucoside dimer ${ }^{1}$ & 9.81 & 851.2511 & & $0.14 \pm 0.00^{\mathrm{a}}$ & $0.02 \pm 0.00^{b}$ & $0.02 \pm 0.00^{\mathrm{b}}$ \\
\hline 34 & Peonidin-(6coumaroyl)-3-glucoside ${ }^{3}$ & 9.87 & 609.1603 & 301.0716 & $0.94 \pm 0.03^{\mathrm{a}}$ & $0.60 \pm 0.02^{b}$ & $0.42 \pm 0.01^{\mathrm{c}}$ \\
\hline 35 & Malvidin-(6-coumaroyl)-3-glucoside ${ }^{1}$ & 9.92 & 639.1708 & 331.0823 & $10.77 \pm 0.02^{\mathrm{a}}$ & $4.43 \pm 0.01^{\mathrm{b}}$ & $2.31 \pm 0.01^{c}$ \\
\hline 36 & Malvidin-glucoside-vinyl-catechin ${ }^{1}$ & 9.99 & 805.1974 & & $0.16 \pm 0.00^{\mathrm{a}}$ & $0.06 \pm 0.00^{b}$ & $0.14 \pm 0.00^{\mathrm{a}}$ \\
\hline 37 & Acetyl-pinotin A ${ }^{1}$ & 10.19 & 667.1657 & & $0.01 \pm 0.00^{\mathrm{a}}$ & $0.00 \pm 0.00^{\mathrm{b}}$ & $0.01 \pm 0.00^{\mathrm{a}}$ \\
\hline 38 & Malvidin 3-O-glucoside 4-vinylphenol (Pigment A) ${ }^{1}$ & 10.22 & 609.1603 & 447.1079 & $0.64 \pm 0.01^{\mathrm{a}}$ & $0.44 \pm 0.00^{\mathrm{b}}$ & $0.44 \pm 0.00^{b}$ \\
\hline 39 & Catechin-ethyl-malvidin-3-coumaroylglucoside dimer ${ }^{1}$ & 10.33 & 955.2785 & & $0.12 \pm 0.00^{\mathrm{a}}$ & $0.04 \pm 0.00^{\mathrm{b}}$ & $0.10 \pm 0.00^{\mathrm{a}}$ \\
\hline 40 & Malvidin acetyl 3-O-glucoside 4-vinylphenol (Acetyl-pigment A) ${ }^{1}$ & 10.50 & 651.1708 & 447.1076 & $0.38 \pm 0.01^{\mathrm{a}}$ & $0.03 \pm 0.00^{\mathrm{b}}$ & $0.02 \pm 0.00^{\mathrm{b}}$ \\
\hline
\end{tabular}

Abbreviations: Retention time (R.T.). Wine lees (WL). ${ }^{a, b, c}$ Different letters indicate significant differences in the content of each individual phenolic compound between the different WL $\left(p<0.05\right.$; one-way ANOVA). ${ }^{1}$ Tentatively quantified using the calibrating curve of malvidin glucoside. ${ }^{2}$ Tentatively quantified using the calibrating curve of cyaniding rutinoside. ${ }^{3}$ Tentatively quantified using the calibrating curve of peonidin rutinoside.

The sample individual phenolic profile on flavanols, flavonols, phenolic acids, and stilbenes is shown in Table 3. The major compounds in all samples were catechin, epicatechin, procyanidin dimer B2, and procyanidin dimer iso1, with higher levels found in CWL compared to Mazuela and Garnacha WL. Regarding the content of the other phenolic families, the major compounds found were: quercetin and isorhamnetin in the flavonols group, gallic acid in the phenolic acid group, and trans-resveratrol and piceatannol in the stilbene group.

Regarding the anthocyanin composition (Table 4), a total of forty different anthocyanins were identified in the WL with malvidin-3-glucoside $>$ malvidin-(6-acetyl)-3glucoside > malvidin-(6-coumaroyl)-3-glucoside as the major compounds. The content of these three compounds was notably higher in the CWL compared to the other WL.

\subsection{Effect of Different Wine Lees on Blood Pressure in Hypertensive Rats}

The antihypertensive effect of Cabernet, Garnacha, and Mazuela WL was evaluated in SHR rats after an acute oral dose $(5 \mathrm{~mL} / \mathrm{kg} \mathrm{bw})$. SBP and DBP results of this study are shown in Figure 4A,B, respectively. As expected, animals that received water did not show changes in their BP. In contrast, Captopril administration $(50 \mathrm{mg} / \mathrm{kg} \mathrm{bw})$ led to a continuous decrease in the animals' SBP and DBP $2 \mathrm{~h}$ post-treatment. The maximum decreases were observed at $6 \mathrm{~h}(-43.2 \pm 3.9$ and $-47.2 \pm 1.5 \mathrm{mmHg}$ for SBP and DBP, 
respectively). Regarding the WL, only CWL showed an antihypertensive effect on both SBP and DBP in SHR, being their behavior similar to the one observed by Captopril. The maximum decrease in BP was also observed at $6 \mathrm{~h}$ post-administration $(-36.4 \pm 3.4$ and $-38.8 \pm 4.6 \mathrm{mmHg}$ for SBP and DBP, respectively). Initial BP values were recovered at 24 or $48 \mathrm{~h}$ for SBP and DBP, respectively. No significant changes in BP were found between the Garnacha or Mazuela WL groups and water group (Figure 4A,B). CWL were selected according their antihypertensive effect for further studies.
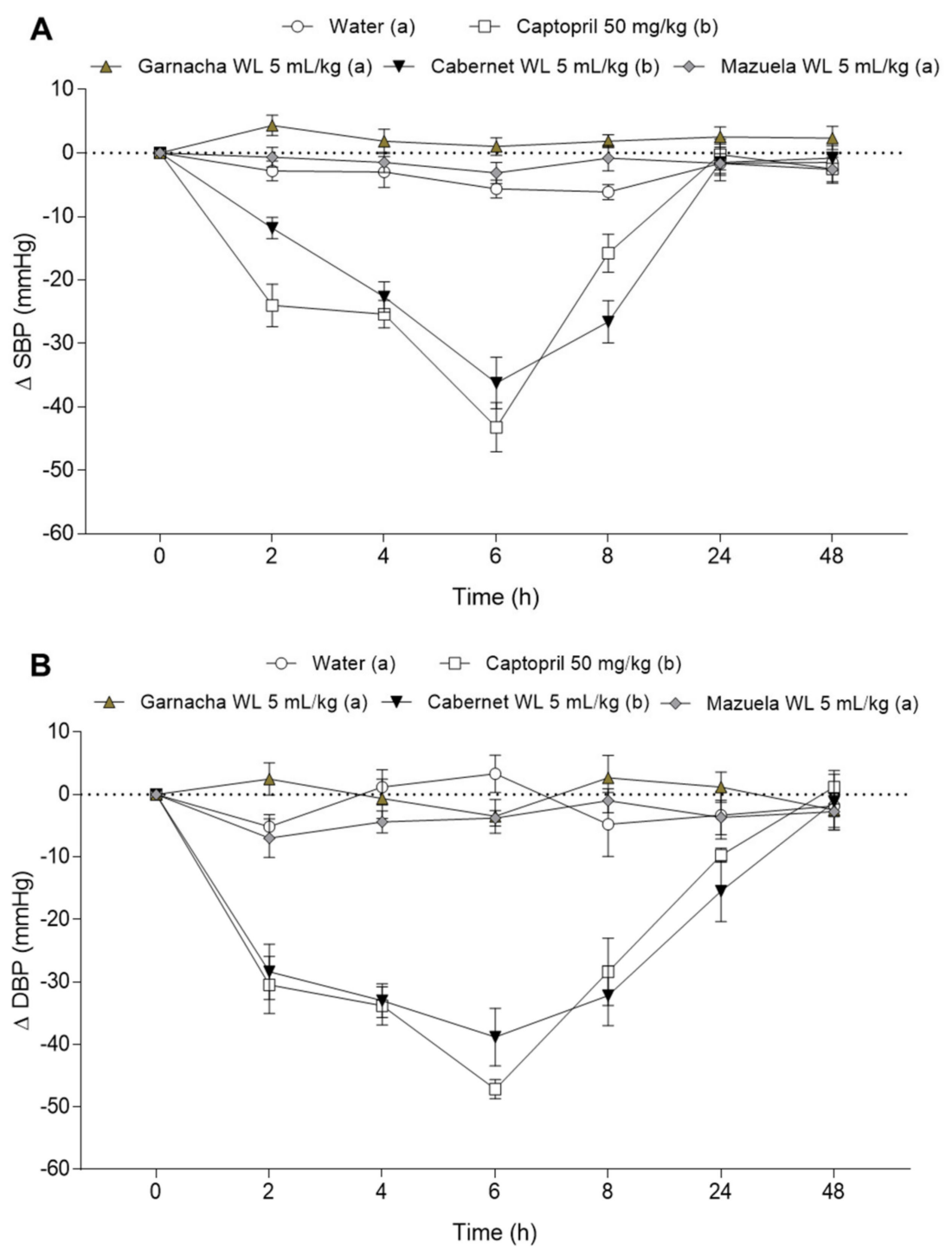

Figure 4. Decrease of systolic blood pressure (SBP, A) and diastolic blood pressure (DBP, B) in spontaneous hypertensive rats after the administration of water, Captopril ( $50 \mathrm{mg} / \mathrm{kg} \mathrm{bw})$, and the three selected wine lees (WL; $5 \mathrm{~mL} / \mathrm{kg}$ bw): Garnacha WL, Cabernet WL, and Mazuela WL. Data are expressed as mean $(n=6) \pm$ SEM. Significant differences $(p<0.05)$ between treatments are represented by different letters in the legend. $p$ value was estimated by two-way ANOVA and Tukey test was used as post hoc.

\subsection{Effect of Cabernet Wine Lees on Blood Pressure in Normotensive Rats}

The effect of CWL on BP was also evaluated in normotensive rats (WKY) in order to discard possible hypotensive effects. Initial values of SBP and DBP were $119.1 \pm 4.2$ and $87.9 \pm 8.8 \mathrm{mmHg}$, respectively. The administration of a single dose of CWL $(5 \mathrm{~mL} / \mathrm{kg} \mathrm{bw})$ did not modify SBP or DBP values in the animals during the experiment (Figure 5). BP values were significantly similar to those showed by the animals that ingested water. 

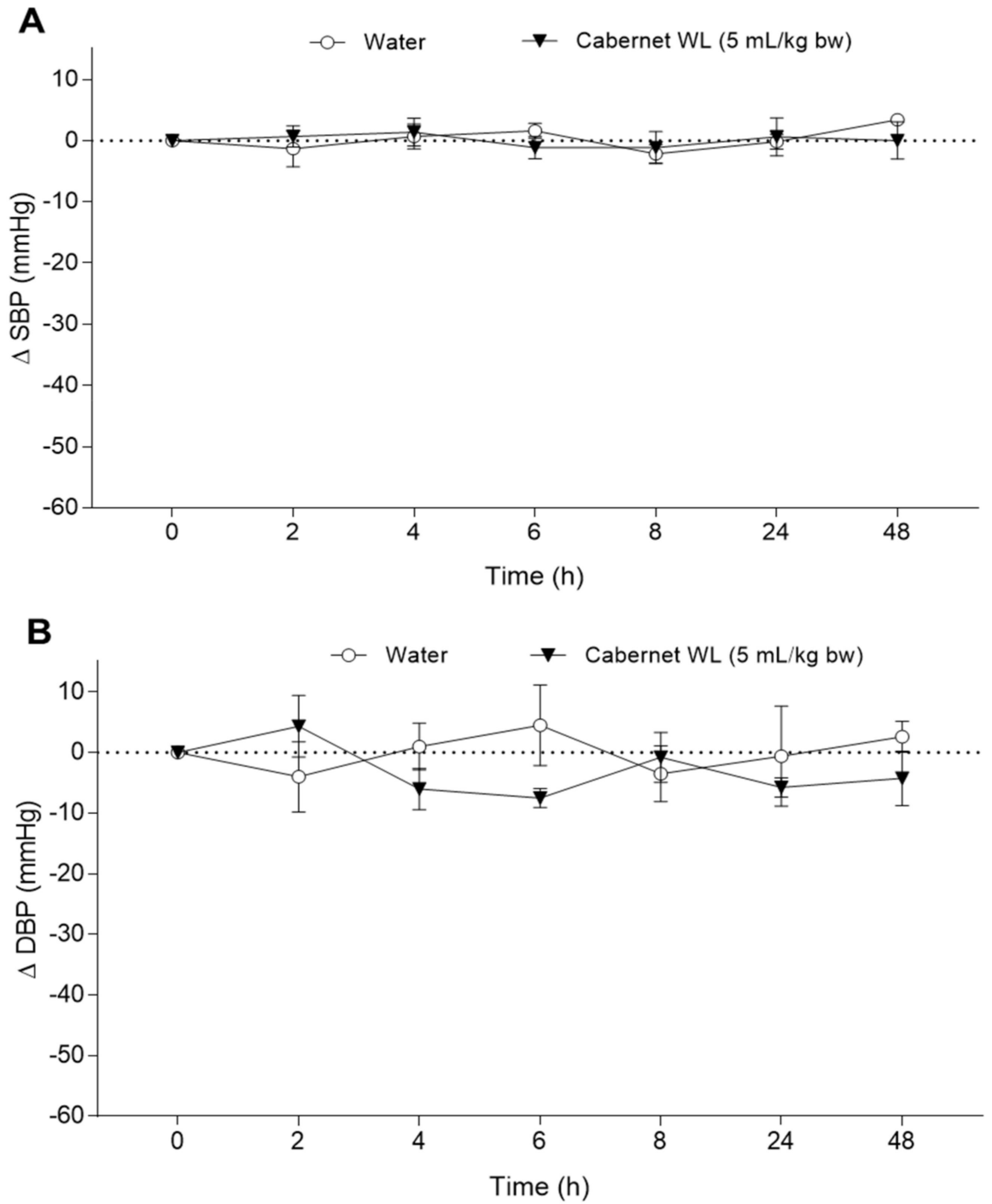

Figure 5. Decrease in systolic blood pressure (SBP, A) and diastolic blood pressure (DBP, B) caused in Wistar-Kyoto rats after the acute administration of water or Cabernet WL ( $5 \mathrm{~mL} / \mathrm{g} \mathrm{bw})$. Data are expressed as mean $(n=6) \pm$ SEM. No significant differences $(p<0.05)$ were found. $p$ was estimated by two-way ANOVA.

\subsection{Variability between Cabernet Wine Lees from Two Different Harvests}

Finally, the variability of ACEi and antihypertensive activities of CWL harvested in two different years (CWL and CWL2) were evaluated. ACEi activity (\%) and IC $_{50}$ did not show differences between CWL from different grape harvests (Figure 6A,B). The antihypertensive properties of CWL and CWL2 were also evaluated in SHR at a single dose of $5 \mathrm{~mL} / \mathrm{kg}$ bw at $6 \mathrm{~h}$ post-administration. No differences were found in SBP and DBP between different harvests (Figure 6C,D). 
A
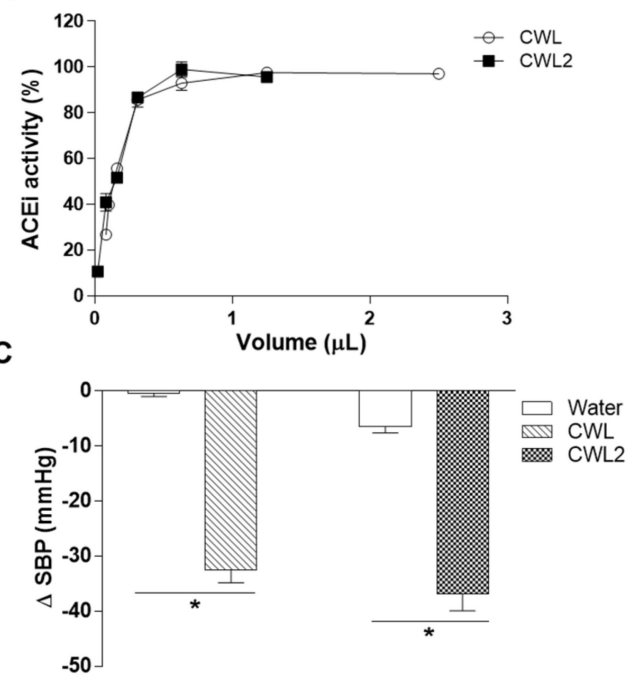

B

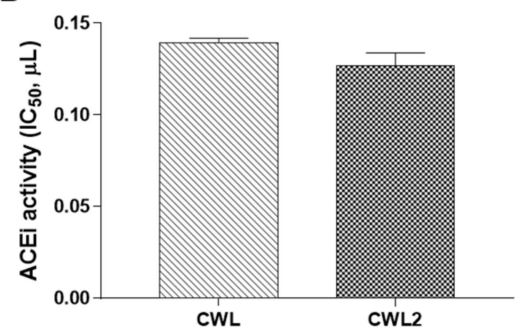

D

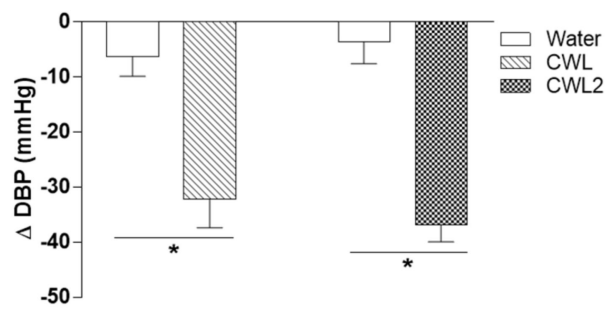

Figure 6. Variability between Cabernet wine lees (CWL) from two different harvests (CWL and CWL2). ACEi activity for both harvests is represented as dose-response curve $(\%, \mathbf{A})$ and $\mathrm{IC}_{50}(\mathbf{B})$. Data are shown as mean $\pm \mathrm{SD}(n=3)$. No significant differences $(p<0.05)$ were found between $\mathrm{IC}_{50}$ values (Student's T-test). Decrease in systolic blood pressure (SBP, C) and diastolic blood pressure (DBP, D) caused in spontaneous hypertensive rats by the acute administration of water, CWL, or CWL2 (both $5 \mathrm{~mL} / \mathrm{kg} \mathrm{bw}$ ). Data are shown as mean $\pm \operatorname{SEM}(n=6)$. No significant differences $(p<0.05)$ were found between CWL and CWL2 (Student's T-test). * represents significant differences $(p<0.05)$ between CWL and CWL2 and their respective water control groups estimated by Student's T-test.

\section{Discussion}

Annually, a large number of agri-food by-products are generated during food processing and their valorization has attracted a great deal of attention over the past few years [9]. In fact, one of the most emerging purposes is to be used as a source of bioactive compounds. These compounds are highly valued by the food, pharmaceutical, and cosmetic industries because they show a wide range of beneficial health effects. In this sense, winery by-products have been successfully used to obtain antioxidant [31], antimicrobial [32], antiinflammatory [33], antihyperglycemic [34], or antihypertensive compounds [14,16,17,35]. For instance, extracts rich in phenolic compounds (proanthocyanidins or resveratrol), with antihypertensive properties in both rats and humans, have been extracted from stem, grape seeds, or skin, respectively [13,15-17,36-38]. However, to our knowledge, no studies have been performed to evaluate whether WL can present ACEi or antihypertensive properties. Thus, the aim of this study was to evaluate the ACEi and antihypertensive activities in several WL. For this, five WL samples were obtained in the elaboration of wine with a single grape variety (red grapes varieties: Cabernet, Garnacha, Mazuela, Merlot, and white grape variety: Macabeo) were selected to determine their ability to inhibit ACE. ACE inhibitors, as Captopril, Enalapril, or Lisinopril, are usually used to treat hypertension [39]. In fact, ACEi activity is commonly used as a screening tool in the search for natural antihypertensive compounds. The determination of the ACEi activity of the five WL showed that lees obtained from red grape varieties exerted higher activity than those obtained from the white grape variety (Table 1; Figure 2). Phenolic compounds are present in red grapes in larger quantities than in white grapes [40]. Therefore, these compounds could be responsible for the ACEi effects since in vitro studies have demonstrated inhibitory properties of phenolic compounds on ACE [8]. Similar results were reported by Pozo-Bayón et al. and AlcaideHidalgo et al., who studied the ACEi activity of red (Tempranillo) and white (Airén, Verdejo and Sauvignon Blanc) wines, respectively [41,42]. The ACEi activity of the WL was also calculated as $\mathrm{IC}_{50}$. The expression of this value in volume is indicative of the microliters of the WL necessary to inhibit the enzyme by $50 \%$ under the assay conditions, where the total volume is $240 \mu \mathrm{L}$. Therefore, it is a measure of the pharmacological potency, given that the 
lower the $\mathrm{IC}_{50}$, expressed in volume, the higher the potency of ACE inhibition of the WL assayed [43]. All WL obtained from red grapes showed a potent ACEi activity, although differences in the $\mathrm{IC}_{50}$ values can be noted (Table 1). ACEi activities of red WL ranged among $0.15 \pm 0.0$ and $0.32 \pm 0.0 \mu \mathrm{L}$. These inhibitory potencies are higher than those reported by other authors in milk fermented with Enterococcus faecalis and Lactobacillus helveticus $[43,44]$. These results clearly reveal important ACEi potency in WL obtained from red grapes. Although ACEi activity is frequently used to select antihypertensive compounds, it does not always correspond to an in vivo effect. Gastrointestinal digestion could produce variations over different WL compounds. In addition, first pass metabolism and microbiota metabolism will significantly modify the ingested phenolic compounds [45]. All these modifications could lead to changes in their ACEi properties. Therefore, in vivo studies must be carried out to demonstrate the antihypertensive effect of WL. In the present study, the antihypertensive effect of Cabernet, Garnacha, and Mazuela WL, selected by their ACEi activity, was evaluated in SHR after a single oral dose of $5 \mathrm{~mL} / \mathrm{kg}$ bw (Figure 4). While Garnacha and Mazuela WL did not exhibit BP-lowering effects, CWL showed a clear antihypertensive effect. The maximum antihypertensive effect was reached at $6 \mathrm{~h}$ post-administration. The ACE inhibitor Captopril also exhibited a similar response time. A maximum decrease in $\mathrm{BP}$ at 4 or $6 \mathrm{~h}$ post-administration has been also reported by our group for other natural ACE inhibitors such as phenolic rich cocoa or grape seed extracts $[30,46]$ or bioactive peptides [47]. Furthermore, the powerful antihypertensive effect of the CWL was similar to that observed for the drug Captopril. Phenolic extracts obtained from grape seeds (GSPE) have shown similar antihypertensive effects, with a maximum drop in BP at $6 \mathrm{~h}$ post-administration $[15,17]$. Similarly, Valls et al. observed a significant enhancement of endothelial function $5 \mathrm{~h}$ after administration to volunteers of a phenolic-enriched olive oil. In addition, this effect correlated with an increase of phenolic-derived metabolites in blood $2 \mathrm{~h}$ after its intake [48]. Notably, the BP-lowering effect produced by CWL (approximately $30 \mathrm{mmHg}$ ) could be a promising result since small reductions in BP may have an important impact on cardiovascular events in the hypertensive population [49]. In this sense, a reduction in $5 \mathrm{mmHg}$ for DBP and $10 \mathrm{mmHg}$ for SBP produces a significant reduction in the risk of suffering or worsening CVD [50,51]. The effect on BP of CWL was also tested in normotensive rats in order to rule out hypotensive effects. CWL did not modify the BP of these animals (Figure 5). This indicates that the antihypertensive effect of these CWL is specific to the hypertensive condition.

In order to understand the different BP-lowering effects exhibited by the tested WL in rats, the phenolic profile of these three samples was studied. Phenolic compounds have been widely investigated due to their large number of beneficial properties, such as their cardioprotective effect [52], in which antihypertensive activity is included [53]. Specifically, a meta-analysis focused on grape phenolic compounds showed that their daily consumption reduced SBP by $1.48 \mathrm{mmHg}$ when compared with the control group [54]. Thus, the different BP-lowering effects exhibited by the three WL would lie within the phenolic composition. According to this, results revealed that CWL contained twice the amount of total phenolic compounds when compared to Mazuela or Garnacha WL. These results highlight the importance of phenolic compounds for the antihypertensive activity of the WL. Specifically, it can be observed a higher concentration of flavanol and anthocyanin families in CWL compared to Mazuela or Garnacha WL (Table 2). Numerous studies have shown that the intake of flavanol-rich foods such as cocoa, red grapes, and red wine can be associated with improved vascular function and can repair and reduce BP in both hypertensive and pre-hypertensive individuals [55]. It has also been reported that flavanol-rich extracts from grape seed or cocoa showed antihypertensive effect after their acute $[15,17,30,46]$ and chronic administration [16] to hypertensive rats. Furthermore, the flavanol monomers epicatechin and catechin have shown antihypertensive effect in both rats and humans administered at low concentrations [56-58]. The high levels of catechin, epicatechin, and procyanidins (Table 3) present in CWL suggest that these compounds could be in part responsible for the BP-lowering effect of this variety of WL. In addition, the 
polyphenol family of anthocyanins has also shown cardioprotective and antihypertensive properties $[59,60]$. Their circulating metabolites have also been directly related to vascular benefits [61]. Moreover, malvidin-3-glucoside, a compound from the anthocyanins family, has been reported as a potent vasodilator [62]. In our study, the anthocyanin content in CWL was higher than in the other WL. The main differences were in the levels of malvidin-3glucoside, malvidin-(6-acetyl)-3-glucoside, and malvidin-(6-coumaroyl)-3-glucoside (Table 4). Therefore, anthocyanin family and specifically these compounds could be also involved in the BP-lowering effect of CWL.

Finally, the ACEi and antihypertensive activities of a CWL coming from a different harvest were also studied. No significant differences in the ACEi activity or in the decrease of BP produced by the different CWL were observed (Figure 6), indicating good reproducibility of the CWL beneficial effects.

\section{Conclusions}

This study shows that WL from red grapes present a potent ACEi activity and that CWL, WL coming from the grape variety Cabernet, also exhibited a potent BP-lowering effect, specific to a hypertensive condition. It is noteworthy that in this study CWL were administered at $5 \mathrm{~mL} / \mathrm{kg}$ bw. This dose corresponds to an intake of $73 \mathrm{~mL} /$ day in humans, using a translation of animal to human doses [63] and estimating the daily intake for an adult human with body weight $70 \mathrm{~kg}$ and body height $175 \mathrm{~cm}$. Although experimental results obtained in animals cannot be directly translatable to humans, the fact that only $73 \mathrm{~mL}$ of $\mathrm{CWL}$ exhibit antihypertensive effects opens the door to the valorization of CWL by their BP-lowering properties. Nevertheless, the quantity of CWL necessary to decrease arterial BP in humans should be definitively established when clinical trials are conducted. CWL antihypertensive activity has been related to their highest content in anthocyanins and flavanols. In addition, these beneficial effects were reproducible in CWL from different vintages. These findings open the door to the use of CWL to alleviate hypertension. At the same time, this study would also allow the wine industry to revalue by-products as $\mathrm{WL}$ and, therefore, reduce their associated environmental problems. However, HTN is a chronic pathology that requires chronic treatment; thus, chronic studies are necessary to evaluate of antihypertensive effect of long-term administration of CWL.

\section{Patents}

Patent application "Wine lees, derivatives thereof and their uses": application number EP20382358.8 and PCT/EP2021/053051.

Author Contributions: Conceptualization, B.M. and F.I.B.; formal analysis, R.L.-F.-S., J.R.S.-R., and M.M.; funding acquisition, B.M., F.I.B., A.A.-A., and M.S.; investigation, R.L.-F.-S., J.R.S.-R., and M.M.; methodology, R.L.-F.-S., J.R.S.-R., and M.M.; supervision, B.M. and F.I.B.; writing-original draft, R.L.-F.-S., B.M., and F.I.B.; writing-review and editing, B.M., F.I.B., A.A.-A., and M.S. All authors have read and agreed to the published version of the manuscript.

Funding: This work has been supported by Grant numbers: RETOS COLABORACIÓN: RTC2017-6044-2 from the Spanish Ministry of Economy and Competitiveness and European Regional Development Fund (FEDER) and AEI-010300-2013-254, AEI-010500-2014-201, and AEI-010500-2015328 from the Spanish Centre for the Development of Industrial Technology (CDTI).

Institutional Review Board Statement: The animal protocols followed in this study were conducted in accordance with the European Communities Council Directive (86/609/EEC) and approved by the Animal Ethics Review Committee for Animal Experimentation of the Universitat Rovira i Virgili and further approved by Generalitat de Catalunya (permission number 10780). 
Acknowledgments: J.R.S.-R. is a recipient of a predoctoral fellowship from the Spanish Ministry of Economy and Competitiveness (Grant number: BES-2017-080919). A.A.-A. and F.I.B. are Serra Húnter Fellows. We would like to thank Niurka Llópiz and Rosa Pastor from the University Rovira i Virgili and $\mathrm{M}^{\mathrm{a}}$ Eugenia Hernández and Irene Cilla from the Cluster Aragonés de Alimentación for their technical support, Antonio del Pino, from the metabolomic facility of the Centre for Omic Sciences (COS) Joint Unit of the Universitat Rovira i Virgili-Eurecat, for his contribution to mass spectrometry analysis and Grandes Vinos y Viñedos for providing us the WL.

Conflicts of Interest: The authors declare no conflict of interest.

\section{References}

1. Oparil, S.; Acelajado, M.C.; Bakris, G.L.; Berlowitz, D.R.; Cífková, R.; Dominiczak, A.F.; Grassi, G.; Jordans, J.; Poulter, N.R.; Rodgers, A.; et al. Hypertension. Nat. Rev. Dis. Prim. 2018, 4, 18014. [CrossRef]

2. Forouzanfar, M.H.; Liu, P.; Roth, G.A.; Ng, M.; Biryukov, S.; Marczak, L.; Alexander, L.; Estep, K.; Abate, K.H.; Akinyemiju, T.F.; et al. Global burden of hypertension and systolic blood pressure of at least $110 \mathrm{to} 115 \mathrm{mmHg}, 1990$ 2015. JAMA J. Am. Med. Assoc. 2017, 317, 165-182. [CrossRef]

3. Hypertension. Available online: https://www.who.int/news-room/fact-sheets/detail/hypertension (accessed on 22 May 2020).

4. Hedayati, S.S.; Elsayed, E.F.; Reilly, R.F. Non-pharmacological aspects of blood pressure management: What are the data? Kidney Int. 2011, 79, 1061-1070. [CrossRef] [PubMed]

5. Snauwaert, E.; Vande, W.J.; De Bruyne, P. Therapeutic efficacy and safety of ACE inhibitors in the hypertensive paediatric population: A review. Arch. Dis. Child. 2017, 102, 63-71. [CrossRef] [PubMed]

6. Sparks, M.A.; Crowley, S.D.; Gurley, S.B.; Mirotsou, M.; Coffman, T.M. Classical Renin-Angiotensin System in Kidney Physiology. Compr. Physiol. 2014, 4, 1201-1228. [PubMed]

7. Brown, N.J.; Vaughan, D.E. Angiotensin-Converting Enzyme Inhibitors. Circulation 1998, 97, 1411-1420. [CrossRef]

8. Margalef, M.; Bravo, F.I.; Muguerza, B.; Arola-Arnal, A. Natural Angiotensin Converting Enzyme (ACE) Inhibitors with Antihypertensive Properties. In Natural Products Targeting Clinically Relevant Enzymes; Wiley-VCH Verlag GmbH \& Co. KGaA.: Weinheim, Germany, 2017; pp. 45-67.

9. Ben-Othman, S.; Jõudu, I.; Bhat, R. Bioactives from Agri-Food Wastes: Present Insights and Future Challenges. Molecules 2020, 25, 510. [CrossRef] [PubMed]

10. Trigo, J.P.; Alexandre, E.M.C.; Saraiva, J.A.; Pintado, M.E. High value-added compounds from fruit and vegetable by-products -Characterization, bioactivities, and application in the development of novel food products. Crit. Rev. Food Sci. Nutr. 2019, 60, 1388-1416. [CrossRef]

11. Mak, T.M.; Xiong, X.; Tsang, D.C.; Yu, I.K.; Poon, C.S. Sustainable food waste management towards circular bioeconomy: Policy review, limitations and opportunities. Bioresour. Technol. 2020, 297, 122497. [CrossRef]

12. Musee, N.; Lorenzen, L.; Aldrich, C. Cellar waste minimization in the wine industry: A systems approach. J. Clean. Prod. 2007, 15, 417-431. [CrossRef]

13. Vázquez-Garza, E.; Bernal-Ramírez, J.; Jerjes-Sánchez, C.; Lozano, O.; Acuña-Morín, E.; Vanoye-Tamez, M.; Ramos-González, M.R.; Chapoy-Villanueva, H.; Pérez-Plata, L.; Sánchez-Trujillo, L.; et al. Resveratrol Prevents Right Ventricle Remodeling and Dysfunction in Monocrotaline-Induced Pulmonary Arterial Hypertension with a Limited Improvement in the Lung Vasculature. Oxid. Med. Cell. Longev. 2020, 2020, 1841527. [CrossRef]

14. Jia, X.; Zhang, R.; Guo, J.; Yue, H.; Liu, Q.; Guo, L.; Zhang, Q. Resveratrol Supplementation Prevents Hypertension in Hypertensive Pregnant Rats by Increasing Sodium Excretion and Serum Nitric Oxide Level. Int. J. Hypertens. 2020, 2020, 4154010. [CrossRef]

15. Pons, Z.; Guerrero, L.; Margalef, M.; Arola, L.; Arola-Arnal, A.; Muguerza, B. Effect of low molecular grape seed proanthocyanidins on blood pressure and lipid homeostasis in cafeteria diet-fed rats. J. Physiol. Biochem. 2014, 70, 629-637. [CrossRef]

16. Pons, Z.; Margalef, M.; Bravo, F.I.; Arola-Arnal, A.; Muguerza, B. Chronic administration of grape-seed polyphenols attenuates the development of hypertension and improves other cardiometabolic risk factors associated with the metabolic syndrome in cafeteria diet-fed rats. Br. J. Nutr. 2017, 117, 200-208. [CrossRef]

17. Quiñones, M.; Guerrero, L.; Suárez, M.; Pons, Z.; Aleixandre, A.; Arola, L.; Muguerza, B. Low-molecular procyanidin rich grape seed extract exerts antihypertensive effect in males spontaneously hypertensive rats. Food Res. Int. 2013, 51, 587-595. [CrossRef]

18. Pérez-Serradilla, J.; De Castro, M.L. Role of lees in wine production: A review. Food Chem. 2008, 111, 447-456. [CrossRef] [PubMed]

19. Romero-Díez, R.; Rodríguez-Rojo, S.; Cocero, M.J.; Duarte, C.M.M.; Matias, A.A.; Bronze, M.R. Phenolic characterization of aging wine lees: Correlation with antioxidant activities. Food Chem. 2018, 259, 188-195. [CrossRef] [PubMed]

20. Jara-Palacios, M.J. Wine Lees as a Source of Antioxidant Compounds. Antioxidants 2019, 8, 45. [CrossRef]

21. Landeka Jurčević, I.; Dora, M.; Guberović, I.; Petras, M.; Rimac Brnčić, S.; Đikić, D. Wine Lees Polyphenols as a Novel Functional Bioactive Compound in the Protection against Oxidative Stress and Hyperlipidemia. Food Technol. Biotechnol. 2017, 55, 109. [CrossRef] [PubMed]

22. De Iseppi, A.; Lomolino, G.; Marangon, M.; Curioni, A. Current and future strategies for wine yeast lees valorization. Food Res. Int. 2020, 137, 109352. [CrossRef] 
23. Romero-Díez, R.; Matos, M.; Rodrigues, L.; Bronze, M.R.; Rodríguez-Rojo, S.; Cocero, M.; Matias, A. Microwave and ultrasound pre-treatments to enhance anthocyanins extraction from different wine lees. Food Chem. 2019, 272, 258-266. [CrossRef] [PubMed]

24. Matos, M.S.; Romero-Díez, R.; Álvarez, A.; Bronze, M.R.; Rodríguez-Rojo, S.; Mato, R.B.; Cocero, M.J.; Matias, A.A. PolyphenolRich Extracts Obtained from Winemaking Waste Streams as Natural Ingredients with Cosmeceutical Potential. Antioxidants 2019, 8, 355. [CrossRef] [PubMed]

25. De La Torre, M.P.D.; Priego-Capote, F.; De Castro, M.D.L. Characterization and Comparison of Wine Lees by Liquid Chromatography-Mass Spectrometry in High-Resolution Mode. J. Agric. Food Chem. 2015, 63, 1116-1125. [CrossRef]

26. Barcia, M.T.; Pertuzatti, P.B.; Rodrigues, D.; Gómez-Alonso, S.; Hermosín-Gutíerrez, I.; Godoy, H.T. Occurrence of low molecular weight phenolics in Vitis vinifera red grape cultivars and their winemaking by-products from São Paulo (Brazil). Food Res. Int. 2014, 62, 500-513. [CrossRef]

27. Mas-Capdevila, A.; Iglesias-Carres, L.; Arola-Arnal, A.; Suarez, M.; Muguerza, B.; Bravo, F.I. Long-term administration of protein hydrolysate from chicken feet induces antihypertensive effect and confers vasoprotective pattern in diet-induced hypertensive rats. J. Funct. Foods 2019, 55, 28-35. [CrossRef]

28. Iglesias-Carres, L.; Mas-Capdevila, A.; Sancho-Pardo, L.; Bravo, F.I.; Mulero, M.; Muguerza, B.; Arola-Arnal, A. Optimized extraction by response surface methodology used for the characterization and quantification of phenolic compounds in whole red grapes (Vitis vinifera). Nutrients 2018, 10, 1931. [CrossRef]

29. Laitila, J.E.; Suvanto, J.; Salminen, J.P. Liquid chromatography-tandem mass spectrometry reveals detailed chromatographic fingerprints of anthocyanins and anthocyanin adducts in red wine. Food Chem. 2019, 294, 138-151. [CrossRef]

30. Quinones, M.; Miguel, M.; Muguerza, B.; Aleixandre, A. Effect of a cocoa polyphenol extract in spontaneously hypertensive rats. Food Funct. 2011, 2, 649-653. [CrossRef]

31. Costa, G.N.S.; Tonon, R.V.; Mellinger-Silva, C.; Galdeano, M.C.; Iacomini, M.; A Santiago, M.C.P.; Almeida, E.L.; Freitas, S.P. Grape seed pomace as a valuable source of antioxidant fibers. J. Sci. Food Agric. 2019, 99, 4593-4601. [CrossRef] [PubMed]

32. Mattos, G.N.; Tonon, R.V.; Furtado, A.A.; Cabral, L.M. Grape by-product extracts against microbial proliferation and lipid oxidation: A review. J. Sci. Food Agric. 2017, 97, 1055-1064. [CrossRef]

33. Wang, H.; Xue, Y.; Zhang, H.; Huang, Y.; Yang, G.; Du, M.; Zhu, M.-J. Dietary grape seed extract ameliorates symptoms of inflammatory bowel disease in IL10-deficient mice. Mol. Nutr. Food Res. 2013, 57, 2253-2257. [CrossRef]

34. Hernández-Salinas, R.; Decap, V.; Leguina, A.; Cáceres, P.; Perez, D.; Urquiaga, I.; Iturriaga, R.; Velarde, V. Antioxidant and antihyperglycemic role of wine grape powder in rats fed with a high fructose diet. Biol. Res. 2015, 48, 53. [CrossRef] [PubMed]

35. Diebolt, M.; Bucher, B.; Andriantsitohaina, R. Wine Polyphenols Decrease Blood Pressure, Improve NO Vasodilatation, and Induce Gene Expression. Hypertension 2001, 38, 159-165. [CrossRef] [PubMed]

36. De Moura, R.S.; Viana, F.S.C.; Souza, M.A.V.; Kovary, K.; Guedes, D.C.; Oliveira, E.P.B.; Rubenich, L.M.S.; Carvalho, L.C.R.M.; Oliveira, R.M.; Tano, T.; et al. Antihypertensive, vasodilator and antioxidant effects of a vinifera grape skin extract. J. Pharm. Pharmacol. 2002, 54, 1515-1520. [CrossRef]

37. Lin, Y.S.; Lu, Y.L.; Wang, G.J.; Chen, L.G.; Wen, C.L.; Hou, W.C. Ethanolic extracts and isolated compounds from small-leaf grape (Vitis thunbergii var. taiwaniana) with antihypertensive activities. J. Agric. Food Chem. 2012, 60, 7435-7441. [CrossRef]

38. Gupta, M.; Dey, S.; Marbaniang, D.; Pal, P.; Ray, S.; Mazumder, B. Grape seed extract: Having a potential health benefits. J. Food Sci. Technol. 2019, 57, 1205-1215. [CrossRef] [PubMed]

39. Tzakos, A.G.; Naqvi, N.; Comporozos, K.; Pierattelli, R.; Theodorou, V.; Husain, A.; Gerothanassis, I.P. The molecular basis for the selection of captopril cis and trans conformations by angiotensin I converting enzyme. Bioorganic Med. Chem. Lett. 2006, 16, 5084-5087. [CrossRef] [PubMed]

40. Mattivi, F.; Guzzon, R.; Vrhovsek, U.; Stefanini, A.M.; Velasco, R. Metabolite Profiling of Grape: Flavonols and Anthocyanins. J. Agric. Food Chem. 2006, 54, 7692-7702. [CrossRef] [PubMed]

41. Alcaide-Hidalgo, J.M.; Martínez-Rodríguez, A.J.; Martin-Álvarez, P.J.; Pueyo, E. Influence of the elaboration process on the peptide fraction with angiotensin I-converting enzyme inhibitor activity in sparkling wines and red wines aged on lees. Food Chem. 2008, 111, 965-969. [CrossRef]

42. Pozo-Bayón, M.A.; Alcaide, J.M.; Polo, M.C.; Pueyo, E. Angiotensin I-converting enzyme inhibitory compounds in white and red wines. Food Chem. 2007, 100, 43-47. [CrossRef]

43. Fuglsang, A.; Nilsson, D.; Nyborg, N.C.B. Cardiovascular effects of fermented milk containing angiotensin-converting enzyme inhibitors evaluated in permanently catheterized, spontaneously hypertensive rats. Appl. Environ. Microbiol. 2002, 68, 3566-3569. [CrossRef]

44. Muguerza, B.; Ramos, M.; Sánchez, E.; Manso, M.; Miguel, M.; Aleixandre, A.; Delgado, M.; Recio, I. Antihypertensive activity of milk fermented by Enterococcus faecalis strains isolated from raw milk. Int. Dairy J. 2006, 16, 61-69. [CrossRef]

45. Iglesias-Carres, L.; Mas-Capdevila, A.; Bravo, F.I.; Aragonès, G.; Arola-Arnal, A.; Muguerza, B. A comparative study on the bioavailability of phenolic compounds from organic and nonorganic red grapes. Food Chem. 2019, 299, 125092. [CrossRef] [PubMed]

46. Pons, Z.; Margalef, M.; Bravo, F.I.; Arola, A.; Muguerza, B. Acute administration of single oral dose of grape seed polyphenols restores blood pressure in a rat model of metabolic syndrome: Role of nitric oxide and prostacyclin. Eur. J. Nutr. 2016, 55, 749-758. [CrossRef] 
47. Mas-Capdevila, A.; Pons, Z.; Aleixandre, A.; Bravo, F.I.; Muguerza, B. Dose-Related Antihypertensive Properties and the Corresponding Mechanisms of a Chicken Foot Hydrolysate in Hypertensive Rats. Nutrients 2018, 10, 1295. [CrossRef]

48. Valls, R.M.; Farràs, M.; Suárez, M.; Fernández-Castillejo, S.; Fitó, M.; Konstantinidou, V.; Fuentes, F.; Lopes-Miranda, J.; Giralt, M.; Covas, M.I.; et al. Effects of functional olive oil enriched with its own phenolic compounds on endothelial function in hyper-tensive patients. A randomised controlled trial. Food Chem. 2015, 167, 30-35. [CrossRef] [PubMed]

49. Turnbull, F. Blood Pressure Lowering Treatment Trialists' Collaboration. Effects of different blood-pressure-lowering regimens on major cardiovascular events: Results of prospectively-designed overviews of randomised trials. Lancet 2003, 362, 1527-1535. [PubMed]

50. Ettehad, D.; Emdin, C.A.; Kiran, A.; Anderson, S.G.; Callender, T.; Emberson, J.; Chalmers, J.; Rodgers, A.; Rahimi, K. Blood pressure lowering for prevention of cardiovascular disease and death: A systematic review and meta-analysis. Lancet 2016, 387, 957-967. [CrossRef]

51. Zanchetti, A. Hypertension: Lower or higher blood-pressure targets for high-risk patients? Nat. Rev. Cardiol. 2016, 13, 637-638. [CrossRef] [PubMed]

52. Dell'Agli, M.; Buscialà, A.; Bosisio, E. Vascular effects of wine polyphenols. Cardiovasc. Res. 2004, 63, 593-602. [CrossRef]

53. Durazzo, A.; Lucarini, M.; Souto, E.B.; Cicala, C.; Caiazzo, E.; Izzo, A.A.; Novellino, E.; Santini, A. Polyphenols: A concise overview on the chemistry, occurrence, and human health. Phytother. Res. 2019, 33, 2221-2243. [CrossRef]

54. Li, S.-H.; Zhao, P.; Tian, H.-B.; Chen, L.-H.; Cui, L.-Q. Effect of Grape Polyphenols on Blood Pressure: A Meta-Analysis of Randomized Controlled Trials. PLoS ONE 2015, 10, e0137665. [CrossRef] [PubMed]

55. Al-Dashti, Y.A.; Holt, R.R.; Stebbins, C.L.; Keen, C.L.; Hackman, R.M. Dietary Flavanols: A Review of Select Effects on Vascular Function, Blood Pressure, and Exercise Performance. J. Am. Coll. Nutr. 2018, 37, 553-567. [CrossRef]

56. Alañón, M.; Castle, S.; Serra, G.; Lévèques, A.; Poquet, L.; Actis-Goretta, L.; Spencer, J. Acute study of dose-dependent effects of (-)-epicatechin on vascular function in healthy male volunteers: A randomized controlled trial. Clin. Nutr. 2020, 39, 746-754. [CrossRef]

57. Rodriguez-Mateos, A.; Weber, T.; Skene, S.S.; Ottaviani, J.I.; Crozier, A.; Kelm, M.; Schroeter, H.; Heiss, C. Assessing the respective contributions of dietary flavanol monomers and procyanidins in mediating cardiovascular effects in humans: Randomized, controlled, double-masked intervention trial. Am. J. Clin. Nutr. 2018, 108, 1229-1237. [CrossRef]

58. Quiñones, M.; Margalef, M.; Arola-Arnal, A.; Muguerza, B.; Miguel, M.; Aleixandre, A. The blood pressure effect and related plasma levels of flavan-3-ols in spontaneously hypertensive rats. Food Funct. 2015, 6, 3479-3489. [CrossRef]

59. Jennings, A.; Welch, A.A.; Fairweather-Tait, S.J.; Kay, C.; Minihane, A.-M.; Chowienczyk, P.; Jiang, B.; Cecelja, M.; Spector, T.; MacGregor, A.; et al. Higher anthocyanin intake is associated with lower arterial stiffness and central blood pressure in women. Am. J. Clin. Nutr. 2012, 96, 781-788. [CrossRef] [PubMed]

60. Zhu, Y.; Bo, Y.; Wang, X.; Lu, W.; Wang, X.; Han, Z.; Qiu, C. The Effect of Anthocyanins on Blood Pressure. Med. 2016, 95, e3380. [CrossRef]

61. Rodriguez-Mateos, A.; Istas, G.; Boschek, L.; Feliciano, R.P.; Mills, E.C.; Boby, C.; Gomez-Alonso, S.; Milenkovic, D.; Heiss, C. Circulating Anthocyanin Metabolites Mediate Vascular Benefits of Blueberries: Insights from Randomized Controlled Trials, Metabolomics, and Nutrigenomics. J. Gerontol. Ser. A Boil. Sci. Med Sci. 2019, 74, 967-976. [CrossRef] [PubMed]

62. Calfío, C.; Huidobro-Toro, J.P. Potent Vasodilator and Cellular Antioxidant Activity of Endemic Patagonian Calafate Berries (Berberis microphylla) with Nutraceutical Potential. Molecules 2019, 24, 2700. [CrossRef]

63. Reagan-Shaw, S.; Nihal, M.; Ahmad, N. Dose translation from animal to human studies revisited. FASEB J. 2007, $22,659-661$. [CrossRef] [PubMed] 